\title{
LA INVESTIGACIÓN NARRATIVA EN LA EDUCACIÓN FÍSICA Y EL DEPORTE
}

\author{
A INVESTIGAÇÃO NARRATIVA NA EDUCAÇÃO FÍSICA E NO ESPORTE
}

NARRATIVE RESEARCH IN PHYSICAL EDUCATION AND SPORT

José Devís-Devís*

Palabras clave:

Educación física. Narrativas.

Deportes.
Resumen: Este artículo muestra sucintamente las principales críticas y potencialidades de la investigación narrativa en el campo de la educación física y el deporte. También se presentan los estudios desarrollados hasta la actualidad en este campo. En primer lugar, aquéllos que han puesto el énfasis en las voces y las experiencias de determinados colectivos marginados hasta ese momento. Posteriormente, se presentan los estudios que enfatizan las historias, las maneras de contarlas y las consecuencias que tienen para quienes las cuentan. También se repasan las principales contribuciones en lengua portuguesa y española. El artículo finaliza introduciendo las contribuciones que se presentan en este número especial, destacando sus principales características.

Palavras chave Educação Física. Narrativas. Esportes.
Resumo: Este artigo mostra sucintamente as principais críticas e potenciais de pesquisa narrativa no campo da educação física e desporto. Os estudos desenvolvidos também são apresentados até à data neste campo. Em primeiro lugar, aqueles que colocaram a ênfase nas vozes e experiências de grupos particulares até então marginalizados. Posteriormente, estudos que enfatizam as histórias, as maneiras de contar e as consequências para aqueles que contam as histórias. As principais contribuições em Português e Espanhol também são revistas. $O$ artigo termina com a introdução das contribuições apresentadas nesta edição especial, destacando suas principais características.

\section{Keywords:}

Physical education. Narratives.

Sports.
Abstract: This article succinctly shows the main criticisms and potentialities of narrative research in the field of physical education and sport. It also presents the studies developed to date in this field. Firstly, those contributions who have emphasized the voices and experiences of certain groups that have been marginalized up to that point. Subsequently, studies that emphasize the stories, the ways of telling them and the consequences they have for those who tell them. A review of the main contributions in Portuguese and Spanish is also presented. The article ends by introducing the contributions of this special issue, highlighting its main characteristics.
*Universitat de València. Valencia, España.

E-mail:Jose.Devis@uv.es

Recebido em: 11-01-2017 Aprovado em: 17-02-2017

(c) (i) () Licence 


\section{INTRODUCCIÓN}

¿Está de moda la investigación narrativa? La proliferación del término 'narrativa' en el ámbito académico, político y de los medios de comunicación, la convierte en una palabra popular y podría considerarse que está de moda, tal y como han manifestado Squire, Andrews y Tamboukou (2008). Sin embargo, la investigación narrativa no parece estar tan de moda, al menos en la educación física y el deporte. Más bien se trata de un tipo de investigación relativamente novedosa en este ámbito concreto y, probablemente por ello, se ha malentendido y descalificado. En unas ocasiones se la ha minusvalorado por considerarla simples historias sin capacidad para iluminar y hacer comprensible las prácticas sociales, tales como la educación física y el deporte (ver SPARKES, 1994). En otras se ha dicho que no aportan nada nuevo y son trabajos carentes de rigor (recogido por DOWLING; GARRETT; LISAHUNTER; WRENCH, 2015). También que tienen un problema de credibilidad y que, en el caso de la enseñanza, las narrativas tienen más que ver con la pedagogía que con la investigación (DOYLE, 1997). Incluso algún investigador cualitativo ha calificado ciertas formas postmodernas de investigación narrativa de artificiosas, narcisistas y de escaso valor para la comprensión de la vida contemporánea y el cambio social efectivo (SANDERS, 1995). Pero la existencia de investigaciones narrativas particulares que merezcan esos calificativos peyorativos, tal y como ocurre en cualquier otro tipo de pesquisa, no puede generalizarse al conjunto de la investigación de su misma clase. Hacer buena investigación narrativa resulta tan difícil y costoso como hacer buena investigación no narrativa. Actualmente, este tipo de pesquisa está admitida como una tradición dentro de la investigación cualitativa en las ciencias sociales que ha ganado fuerza durante las últimas décadas y enriquece el corpus de conocimiento del ámbito de la educación física y el deporte (SMITH; SPARKES, 2008a, 2009).

En un artículo publicado hace unos años en esta misma revista, Pérez-Samaniego, Devís-Devís, Smith y Sparkes (2011) presentaron las principales características y la utilidad de la investigación narrativa para nuestro campo. Por ejemplo, esta investigación se justifica porque contar historias es consubstancial a nuestra naturaleza humana (MACINTYRE, 1981; JOSSELSON, 2006), porque las personas vivimos vidas sociales susceptibles de ser contadas (SARBIN, 1986; POLKINGHORNE, 1988) y porque la subjetividad emerge como preocupación especial en sociedades cambiantes e inestables que hacen extremadamente vulnerables a las personas (LIEBLICH; TUVAL-MASHIACH; ZILBER, 1998). Su contribución más singular reside en que los relatos de las personas son a la vez individuales y sociales, conectan las identidades personales con la cultura en la que se construyen y las vidas particulares se vinculan con las estructuras sociales (SPARKES, 2002). Es decir, la investigación narrativa permite analizar la complejidad, la singularidad, las interacciones y las sofisticadas maneras en que las personas dan sentido a la vida, a las historias y al mundo que les rodea (DENZIN, 2010). En suma, este tipo de investigación se ocupa de utilizar historias que vale la pena contar, que enriquecen la comprensión humana, social y cultural, y que cuentan con un propósito moral o político para un cambio social positivo (CHATAIKA, 2005).

\section{LA INVESTIGACIÓN NARRATIVA: UN BREVE RECORRIDO}

Los inicios de la investigación narrativa en el ámbito de la educación física y el deporte se encuentran en las historias de vida del profesorado de educación física que se ocupaban 
de explorar y comprender sus carreras profesionales y ciclos vitales (SIKES, 1988; SPARKES; TEMPLIN, 1992; TEMPLIN; SPARKES; GRANT; SCHEMPP, 1994; TEMPLIN; SPARKES; SCHEMPP, 1991), sus condiciones de trabajo (MACDONALD, 1999; MACDONALD; KIRK, 1996) y sus identidades profesionales (ARMOUR, 1997; DOWLING-N/ESS, 1996), de género y sexualidad (CLARKE, 1997; SPARKES, 1994; SQUIRES; SPARKES, 1996; SYKES, 1998). Estos primeros estudios, centrados en las experiencias del profesorado, se extendieron al alumnado escolar (HICKEY; FITZCLARENCE, 1999; OLIVER, 1999) y universitario (DEVÍSDEVÍS; SPARKES, 1999). Con ello buscaban amplificar las voces de colectivos marginados, silenciados o poco atendidos hasta entonces, así como conocer las experiencias subjetivas que permitían iluminar las razones de las actuaciones de estas personas y colectivos.

La investigación narrativa que se ocupa de las voces de colectivos discriminados ha seguido desarrollándose posteriormente (BERG SVENDBY; DOWLING, 2012; FITZGERALD; STRIDE, 2012; GROVES; LAWS, 2003; MAIVORSDOTTER; LUNDVALL, 2009; RICH, 2004; SYKES; GOLDSTEIN, 2004; WRENCH; GARRETT, 2012). Pero también se observa un interés creciente por estudios auto-narrativos que se ocupan de experiencias subjetivas diversas y de corporalidades individuales. Este nuevo tipo de estudios abordan formas novedosas de representar la investigación narrativa como son la autoetnografía, la ficción etnográfica y otras formas de pesquisa narrativa del cuerpo y los sentidos. Esta evolución se observa muy bien en los trabajos pioneros de Sparkes $(1996,1997,1999)$ que exploraron, precisamente, estos caminos investigativos y prepararon el tránsito a estudios recientes con características substancialmente diferentes. El énfasis en las voces y las experiencias de determinados colectivos va dando paso al interés por las propias historias, las maneras de contarlas y las consecuencias que tienen en las vidas de quienes las cuentan. Este cambio de énfasis ha estimulado la emergencia de nuevas aproximaciones a la manera de obtener y representar el conocimiento que han enriquecido nuestro campo (p.e. ALLEN COLLINSON, 2008; BOSSLE; MOLINA; KREUGSBURG-MOLINA, 2014; CARLESS, 2012; DENISON, 1996; FITZGERALD, 2007; GONZÁLEZ-CALVO; BARBERO-GONZÁLEZ; BORES-CALLE; MARTÍNEZ-ÁLVAREZ, 2014; PHOENIX; SPARKES, 2006; SICILIA; FERNÁNDEZ-BALBOA, 2006; SMITH; SPARKES, 2002; SPARKES; DOUGLAS, 2007; SPARKES; NILGES; SWAN; DOWLING, 2003; SMITH; SPARKES, 2008b). De acuerdo con Squire, Andrews y Tamboukou (2008), los nuevos estudios ven las historias y narrativas como códigos construidos dialógicamente y no como expresión de estados internos de las personas a las que se les permite dar voz y recoger sus experiencias. Son estudios influidos por el postmodernismo que se preocupan menos por las experiencias de las personas que por deconstruirlas, es decir, por otra manera de preocuparse de ellas. Desde esta perspectiva se considera importante el momento, el contexto y la audiencia particular en que toman forma concreta las narrativas. También se interesan por cómo las maneras de contar los relatos ejemplifican estructuras narrativas de carácter cultural más amplio. En definitiva, las investigaciones del primer tipo tienen un carácter más realista porque intentan mostrar evidencias empíricas y explicaciones teóricas a la experiencia personal, mientras que las de segundo tipo tienen un carácter relativista porque se reconoce el carácter situado, parcial y subjetivo de las historias, y la experiencia personal adquiere un sentido entre diversas interpretaciones posibles (PÉREZ-SAMANIEGO et al., 2011).

En la actualidad, la investigación narrativa se caracteriza por la diversidad de enfoques que derivan de las maneras que existen de entenderla. Para unos es una perspectiva de investigación y para otros un método, un conjunto de estrategias de escritura, un tipo novedoso 
de investigación cualitativa o, como sugieren Clandinin y Connelly (2000), varias de estas cosas a la vez. Pero esta diversidad no debe entenderse como un problema o un demérito sino, más bien al contrario, un signo de riqueza. Esto es válido para las ciencias sociales en general y para el ámbito particular de la educación física y el deporte, tal y como ha puesto de manifiesto el monográfico reciente de Sport, Education and Society, editado por Dowling y Garrett (2016). Estas autoras se refieren a la existencia de un bricolaje de investigaciones narrativas en nuestro ámbito. Según Gergen y Gergen (2011), este bricolaje es el resultado de tensiones diversas que provienen de las distintas maneras de entender este tipo de investigación. Es decir, el resultado del tira y afloja entre una pesquisa que enfatiza las experiencias personales u otra que enfatiza las historias, una que se centra en una dimensión psicológica o en una dimensión social de los relatos y una forma de investigación que se ocupa de la singularidad o en cambio lo hace de la multiplicidad de narrativas de acuerdo con las audiencias. Estas tensiones muestran el dinamismo internacional existente y, por ello, recomiendan el diálogo entre la pluralidad de perspectivas vigentes. En consecuencia, todos podemos aprender de los demás, compartir ideas y trabajar para encontrar formas de investigación narrativa que permitan obtener nuevo conocimiento y comprender las formas complejas de la vida de las personas en movimiento.

En lengua portuguesa y española también existen investigaciones narrativas del primer tipo, es decir, de las que se preocupan de las voces del profesorado y alumnado en las escuelas, así como en la formación del profesorado de educación física. En particular, se orientan a la construcción de identidades profesionales (BARROSO; CAMARGO, 2015; RODRIGUES; FIGUEIREDO, 2011; SPARKES, 1996), el proceso de construcción de saberes profesionales (BETTI; MIZUKAMI, 1997; GIL; MORENO; FERNÁNDEZ, 2015), la visibilidad de espacios y momentos clave en su formación inicial y permanente (OLIVEIRA; SANTOS; FERREIRA, 2012), el aprendizaje de la incompetencia motriz (GONZÁLEZ-CALVO; BARBERO-GONZÁLEZ; BORES-CALLE; MARTÍNEZ-ÁLVAREZ, 2013), la trayectoria profesional (CÓRDOBA, 2015; FOLLIE; FARIAS; BOSCATTO; NASCIMENTO, 2009; SANTOS; BRACHT; ALMEIDA, 2009) o las memorias comparativas que alrededor de diversos temas presenta el profesorado de educación infantil (MARTÍNEZ-ÁLVAREZ; GARCÍA-MONGE; BORES-CALLE, 2015). Algunas se centran en las creencias que los estudiantes universitarios tienen sobre la educación física escolar (LÓPEZ-PASTOR; GARCÍA PEÑUELA; PÉREZ-BRUNICARDI; LÓPEZ-PASTOR; MONJAS AGUADO, 2004), las experiencias evaluativas en formación inicial (SANTOS; MAXIMIANO, 2013), la complejidad de dicha formación (MATTOS; PRADOS; PADUA, 2013) y el lenguaje que utilizan los estudiantes universitarios sobre la masculinidad (CANO; ABAD, 2015). Por último, algunos estudios se ocupan de las experiencias de los estudiantes de educación física escolar para revalorizar el conocimiento práctico en la asignatura ( $F A B R$; ROSSI; PEREIRA, 2016) o se analizan los discursos identitarios de practicantes de capoeira (ALMEIDA; TAVARES; SOARES, 2012).

Frente a este nutrido grupo de investigaciones apenas se cuenta con unos pocos estudios que sitúan a las historias, la manera de contarlas y sus consecuencias en el centro de sus intereses investigativos. Entre ellos se encuentran dos contribuciones que ejemplifican el valor de la ficción en la investigación social sobre la educación física y el deporte. La primera es la ficción autobiográfica que acomete Barbero-González (2006) porque de esa manera puede reflejar mejor la cultura corporal hegemónica que impregna la vida diaria de una sociedad desarrollada, según sus palabras, de forma más coloquial y cercana a los participantes. La segunda es la ficción etnográfica de Martos-García y Devís-Devís (2015) que condensa, en un 
solo día, mucho de lo que acontece en dos años y medio de trabajo de campo en una prisión. De esta manera pretenden acercar a los lectores y lectoras, la vida cotidiana de la prisión y el papel que juega la actividad física en dicho entorno, facilitando la evocación de emociones e interacciones particulares que se perderían con un relato más convencional. Por último, destacaría los estudios que analizan narrativas deportivas de los medios de comunicación, a caballo entre el deporte y el periodismo. Por ejemplo, el análisis del discurso deportivo en los magacines radiofónicos que apunta a una especie de des-deportivización (ÁLVAREZ-VILLA, 2001) o cómo el discurso televisivo del fútbol femenino influye en el campo de juego (MOURÃO; MOREL, 2005) o cómo éste discurso del fútbol femenino se disloca en uno propiamente deportivo y otro estético que enfatiza una visión esencialista del género femenino (SANTOS; MEDEIROS, 2012).

\section{NUEVAS APORTACIONES Y COMENTARIOS FINALES}

La reducida representación de investigaciones narrativas en lengua española y portuguesa que se centran en las historias, los textos y los discursos, sirve para justificar el espacio especial que dedica la revista Movimento a este tipo de pesquisa. Las investigaciones que se presentan en este monográfico son ejemplos de las nuevas tendencias, especialmente de las que enfatizan la dimensión subjetiva de los participantes, ya sean sujetos o investigadores, las historias que se cuentan, la manera de contarlas y sus efectos o varias de estas cosas a la vez. Así tenemos que los artículos de Caddick y Smith y de Monforte y Pérez-Samaniego se centran en un tema de interés cada uno, un contenido sobre el que indagan para profundizar en las experiencias subjetivas de los participantes, veteranos de guerra en el primer caso y estudiantes de educación física en el segundo.

En concreto, el artículo de Caddick y Smith se ocupa de las vivencias de unos soldados veteranos que hacen surf para sobrellevar las heridas físicas, personales y sociales de la guerra. Si bien los autores presentan una amplia riqueza de experiencias de los participantes mientras hacen esta práctica física, sus historias revelan cómo se conecta lo subjetivo con lo social y cultural y cómo experimentan sus nuevas corporalidades en el surf y dan sentido a lo que viven. Esto lo hacen con tres tipos de historias colectivas de tipo realista que deriva de un análisis dialógico narrativo del conjunto de experiencias de los veteranos. Una se centra en las sensaciones físicas del surf y cómo les ayuda en sus vidas. Otra se ocupa del sentimiento de identidad colectiva y cómo esto les ayuda a superar sus problemas de salud y bienestar. Y la tercera lo hace sobre las maneras de canalizar su sufrimiento y sus problemas como hombres que adoptan diversas versiones de masculinidad aceptable. Estas tres historias las construyen a partir de la idea de Frank (2010) que considera a las historias no sólo como simples representaciones pasivas de lo que les acontece a las personas sino como entes actuantes que también tienen consecuencias para sus vidas.

El trabajo de Monforte y Pérez-Samaniego escudriña en las experiencias del miedo desde una perspectiva socio-relacional que las sitúa en un entorno personal, social y cultural concreto de la educación física escolar en el que se evidencian particulares relaciones sociales de poder. Para ello construyen la historia de Eva mediante estrategias narrativas de ficción, las prácticas analíticas creativas que denomina Richardson (2000), con las que integran el qué y el cómo de la historia. En particular, utilizan los datos etnográficos para ser fieles a 
Ios acontecimientos y las voces recogidas, pero dando forma a los ambientes y rellenando los huecos de la historia con estrategias de ficción creativa que hace del relato una historia reconocible por los lectores y lectoras. Aunque se centra en la historia de Eva, también es la historia de otros estudiantes, chicos y chicas, de su misma clase que tienen el mismo profesor de educación física. En este sentido, la historia es personal y social a la vez y trata de un tema, el miedo, que culturalmente conecta con una asignatura que forma parte de la escolarización obligatoria.

Las aportaciones de Martos-García y Devís-Devís y Lozano-Sufrategui y Carless no se centran en un contenido o temática particular como ocurría en los dos artículos anteriores sino que convierten el proceso de investigación narrativa en su propio objeto de interés. La primera de ellas cuenta el proceso seguido por el investigador principal y el amigo crítico, así como lo que les rodea a lo largo de la investigación realizada en el polideportivo de una prisión. Es decir, con los datos de un estudio previo construyen una historia de no-ficción de sí mismos, una autoetnografía, en la que el análisis de los datos se encuentra en la propia historia, como sugiere Richardson (1994).

El trabajo de Lozano-Sufrategui y Carless se centra en los problemas, dilemas y dificultades de una investigadora formada desde un paradigma positivista que se embarca por primera vez en una investigación narrativa. La forma en que plasman las reflexiones en torno a los avatares de su proceso es un relato confesional coherente con el contenido de lo que cuentan. Dicho relato está formado por los momentos clave en la trayectoria de la investigadora, en interacción con su supervisor, y por ello le denominan etnografía dual. De esta manera, retratan cómo evoluciona el pensamiento de la primera autora y las maneras de encarar los retos que se le presentan en su proceso de aprendizaje investigador.

Con estas dos últimas aportaciones se ayuda a nuevos y viejos investigadores y a sus directores o supervisores académicos en el aprendizaje de la investigación narrativa mediante la visibilidad de los entresijos y dilemas de su desarrollo como actividad práctica. Como señalaba Ball (1990) al referirse a la investigación cualitativa, podemos decir que la investigación narrativa no se aprende mediante el uso de técnicas e instrumentos de pesquisa sino que se aprende investigando. Por ello, estos trabajos confesionales resultan de utilidad inspiradora, además de dotar de reflexividad y trascendencia política al conjunto del proceso de investigación (ALEXANDER, 2011). Hacer explícito dicho proceso es un elemento clave para la transparencia y el rigor metodológico, un importante recurso ético y una forma de autocrítica y fortalecimiento de las pesquisas narrativas. También debemos añadir la descripción del papel jugado por el azar, las casualidades y otras circunstancias particulares y personales que tienen lugar antes, durante y después del proceso de la investigación. De esta manera, los lectores y lectoras podrán hacerse una idea equilibrada del producto final y juzgar por sí mismas el trabajo en cuestión.

Por último, contamos con un artículo de reflexión teórico-metodológica sobre el uso de la investigación narrativa para construir relatos históricos del profesorado que contrarresten ('a contrapelo' que diría Benjamin, 2008) las narrativas históricas oficiales o las grandes narrativas neoliberales que se están imponiendo actualmente en Brasil y en muchos otros lugares. En este ensayo, Lopes, Wittizorecki y Molina proponen que esta pesquisa sirva para visibilizar narrativas de 'asedio', que dicen 'no' a las políticas y reformas de retroceso en la educación y la educación física. Estas narrativas se asemejan a la presentada por Raimundo 
Silva, el personaje central de la novela de José Saramago 'Historia del cerco de Lisboa' que con un simple 'no' cambia el sentido de la historia del sitio de la ciudad para liberarla de los moros en el siglo XII. Esta estimulante reflexión sobre el papel de la investigación narrativa conecta con una propuesta anterior del mismo grupo de investigación que, además de realizar investigación narrativa (BOSSLE; MOLINA; KREUSBURG-MOLINA, 2014), defienden su uso en la investigación de la educación física (WITTIZORECKI et al., 2006).

Confío en que esta muestra de trabajos de investigación narrativa seduzca a los lectores y lectoras y potenciales investigadores del campo de la educación física y el deporte. Con ella se abren nuevas posibilidades para indagar en dimensiones, entresijos y encrucijadas de la vida personal, social y cultural de los sujetos en movimiento que, hasta hace poco tiempo, resultaban insospechadas para muchos de nosotros por falta de nuevas perspectivas y estrategias metodológicas disponibles a nuestro alcance.

\section{REFERENCIAS}

ALEXANDER, Bryant. K. Standing in the wake: A critical auto/ethnographic exercise on reflexivity in three movements. Cultural Studies $\leftrightarrow$ Critical Methodologies, v.11, n.2, 98-107, 2011.

ALLEN-COLLINSON, Jacquelyn. Running the routes together: corunning and knowledge in action. Journal of Contemporary Ethnography, v.37, n.1, p. 38-61, 2008.

ALMEIDA, Juliana A.; TAVARES, Otàvio; SOARES, Antonio J.. A reflexividade nos discursos identitários da capoeira. Revista Brasileira de Ciências do Esporte, v. 34, n. 2, p. 375-390, 2012.

ÁLVAREZ-VILLA, Ángeles. Deporte y pasiones comunicativas: el caso del magacín radiofónico español. In: DEVÍS-DEVÍS, José (coord.) La educación física, el deporte y la salud en el siglo XXI. Alcoy: Marfil, 2001. p. 245-267.

ARMOUR, Kathleen M.. Developing a personal philosophy on the nature and purpose of physical education: Life history reflections. European Physical Education Review, v.3, n.1, p. 68-82, 1997.

BALL, Stephen J. Self doubt and soft data: social and technical trajectories in ethnographic fieldwork. In: ALLEN, JoBeth.; GOETZ, Judith P. Qualitative research in education. Teaching and learning qualitative traditions. Athens: University of Georgia, 1990. p. 200-221.

BARBERO, José I. Ficción autobiográfica en torno a la cultura corporal y la vida cotidiana. Educación Física y Deporte, v. 25, n.2, p. 47-63, 2006.

BARROSO, Felipe; CAMARGO, Maria C. Formação da identidade profissional de professoras de educação física: um olhar a partir das narrativas biográficas. Revista Eletrônica Pesquiseduca, v. 7, n. 14, p. 452- 469, 2015.

BENJAMIN, Walter. Tesis sobre la historia y otros fragmentos. México: Ítaca-UACM, 2008.

BERG SVENDBY, Ellen; DOWLING, Fiona. Negotiating the discursive spaces of inclusive education: Narratives of experience from contemporary Physical Education. Scandinavian Journal of Disability Research, v.15, n.4, p. 361-378, 2012. 
BETTI, Irene C.; MIZUKAMI, Maria G. História de vida: trajetória de uma professora de Educação Física. Motriz, v. 3, n. 2, p.108-115, 1997.

BOSSLE, Fabiano; MOLINA, Vicente; KREUSBURG MOLINA, Rosane. The eye of the hurricane: autoethnography in the southern Brazilian school context. International Journal of Qualitative Studies in Education, v.27, n.10, p. 1226-1242, 2014.

CANO, Juliá A.; ABAD, Johnathan S. Formación de maestros en educación física: un trasegar hacia un amanera más sensible de vivir la masculinidad. VIREF-Revista de Educación Física, v.4, n.3, p. 87-101, 2015.

CARLESS, David. Negotiating sexuality and masculinity in school sport: an autoethnography. Sport, Education and Society, v.17, n.5, p. 607-625, 2012.

CHATAIKA, Tsitsi. Narrative research: what's in a story? In: NORDIC NETWORK FOR DISABILITY RESEARCH CONFERENCE NORWAY, 8. 2005. Oslo, $14^{\text {th }}$ and $16^{\text {th }}$ Apr. 2005.

CLANDINNIN, Jean; CONNELLY, Michael. Narrative inquiry : Experience and story in qualitative research. San Francisco: Jossey-Bass, 2000.

CLARKE, Gill. Playing a part: The lives of lesbian physical education teachers. In: Gill CLARKE, Gill; HUMBERSTONE, Barbara (eds.): Researching Women and Sport, London: Macmillan, 1997. p. 36-49.

CÓRDOBA, Txema. La aventura de aprender: Relato autobiográfico del viaje a Ítaca de un docente reflexivo. Retos, n.28, p. 285-290, 2015.

DENISON, Jim. Sport narratives. Qualitative Inquiry, v.2, n.3, p. 351-362, 1996.

DENZIN, Norman. The qualitative manifesto: A call to arms. Walnut St, CA: Left Coast, 2010.

DEVÍS-DEVÍS, José; SPARKES, Andrew C. Burning the book: A biographical study of a pedagogically inspired identity crisis in physical education. European Physical Education Review, v.5, n.2, p. 135-152, 1999.

DOWLING, Fiona; GARRETT, Robyne. Narrative inquiry and research on physical activity, sport and health: exploring current tensions. Sport, Education and Society, v.21, n.1, p. 1-6, 2016.

DOWLING, Fiona; GARRETT, Robyne, LISAHUNTER; WRENCH, Alison. Narrative inquiry in physical education research: the story so far and its future promise. Sport, Education and Society, v.20, n.7, p. 924-940, 2015.

DOWLING-N/ESS, Fiona. Life events and curriculum change: The life history of a Norwegian physical educator. European Physical Education Review, v.2, n.1, p. 41-53, 1996.

DOYLE, Walter. Heard any really good stories lately? A critique of the critics of narrative in educational research. Teaching and Teacher Education, v.13, n.1, p.93- 99, 1997.

FABRI, Eliane; ROSSI, Fernanda; FERREIRA Lilian A. Episódios marcantes das aulas de educação física: valorizando as experiências dos alunos por meio de narrativas. Movimento, $v$. 22, n. 2, p. 583-596, 2016.

FITZGERALD, Hayley. Dramatizing physical education: using drama in research. British Journal of Learning Disabilities, v.35, p. 253-260, 2007. 
FITZGERALD, Hayley; STRIDE, Annette. Stories about physical education from young people with disabilities. International Journal of Disability, Development and Education, v.59, n.3, p. 283-293, 2012.

FOLLE, Alexandra; FARIAS, Gelcemar O.; BOSCATTO, Juliano D.; NASCIMENTO. Juarez V. Construção da Carreira Docente em Educação Física: Escolhas, Trajetórias e Perspectivas. Movimento, v.15, n.1, p. 25-49, 2009.

FRANK, Arthur. Letting stories breathe: A socio-narratology. Chicago: University of Chicago Press, 2010.

GERGEN, Kenneth J.; GERGEN, Mary M. Narrative tension. Perilous and productive. Narrative Inquiry, v.21, n.2, p. 374-381, 2011.

GIL, Karen L.; MORENO, William; FERNANDEZ VAZ, Alexandre. Historia de una práctica profesoral artística en Educación Física: Expresiones del potencial corporal. Estudios Pedagógicos, v.41, n. Especial, p. 67-79, 2015

GONZÁLEZ-CALVO, Gustavo; BARBERO-GONZÁLEZ, José I.; BORES-CALLE, Nicolás; MARTÍNEZ-ÁLVAREZ, Lucio. Relatos autobiográficos sobre la incompetencia motriz aprendida de un docente novel de educación física y sus posibilidades autoformativas. Revista Iberoamericana de Educación, n.62, p. 39-56, 2013.

GONZÁLEZ-CALVO, Gustavo; BARBERO-GONZÁLEZ, José I.; BORES-CALLE, Nicolás; MARTÍNEZ-ÁLVAREZ, Lucio. (Re)construction of a teacher's professional identity from his initial training: Autobiographical narration. The Open Sports Science Journal, v.7, p. 113-120, 2014. Suppl-2, M5.

GROVES, Suzanne; LAWS, Chris. The use of narrative in accessing childrens experiences of physical education. European Journal of Physical Education, v.8, p. 160-174, 2003.

HICKEY, Chris; FITZCLARENCE, Lindsey. Educating boys in sport and physical education: Using narrative methods to develop pedagogies of responsibility. Sport, Education and Society, v.4, n.1, p. 51-62, 1999.

JOSSELSON, Ruthellen. Narrative research and the challenge of accumulating knowledge. Narrative Inquiry, v.16, n.1, p. 3-10, 2006.

LIEBLICH, Amia; TUVAL-MASHIACH, Rivka; ZILBER, Tamar. Narrative research. Reading, analysis and interpretation. London: Sage, 1998.

LÓPEZ PASTOR, Víctor M.; GARCÍA-PEÑUELA, Ana; PÉREZ BRUNICARDI, Darío; LÓPEZ PASTOR, Esther M.; MONJAS AGUADO, Roberto. Las historias de vida en la formación inicial del profesorado de educación física. Revista Internacional de Medicina y Ciencias de la Actividad Física y el Deporte, v.4, n.13, p. 45-57, 2004.

MAIVORSDOTTER, Ninitha; LUNDVALL, Suzanne. Aesthetic experience as an aspect of embodied learning: Stories from physical education student teachers. Sport, Education and Society, v.14, n.3, p. 265-279, 2009.

MACDONALD, Doune. The "professional" work of experienced physical education teachers. Research Quarterly for Exercise and Sport, v.70, n.1, p. 41-54, 1999.

MACDONALD, Doune; KIRK, David. Private lives, public lives: surveillance, identity and self in the work of beginning physical education teachers. Sport, Education and Society, v.1, n.1, p. 59-75, 1996. 
MACINTYRE, Alasdair. After virtue. Notre Dame: University of Notre Dame, 1981.

MARTÍNEZ-ALVAREZ, Lucio; GARCÍA-MONGE, Alfonso; BORES-CALLE, Nicolás. Dentro de la escuela, con la escuela adentro: indagando en el proceso de la propia socialización sobre el acceso al colegio y el cuerpo en el aula a través de un ejercicio de tri-etnografía. Movimento, v. 21, n. 4, p. 945-957, 2015.

MARTOS-GARCÍA, Daniel; DEVIIS-DEVÍS, José. Un día cualquiera en la cárcel: la etnografía-ficción como representación de una investigación. AlBR, Revista de Antropología Iberoamericana, v.10, n.3, p. 355-376, 2015.

MATTO, Berenice; PRADOS, Esther; PADUA, Daniela. La voz del alumnado: Una investigación narrativa acerca de lo que siente, piensa, dice y hace el alumnado de Magisterio de Educación Física en su formación inicial. Movimento, v. 19, n. 4, p. 251-269, 2013.

MOURÃO, Ludmila; MOREL, Marcia. As narrativas sobre o futebol feminino: o discurso da mídia impressa em campo. Revista Brasileira de Ciências do Esporte, v. 26, p. 73-86, 2005.

OLIVEIRA, Aline V.; SANTOS, Wagner; FERREIRA, Amarílio. Tempos de escola: narrativas da formação discente ao ofício docente. Movimento, v. 18, n.3, p. 119-139, 2012.

OLIVER, Kimberly L. Adolescent girls' body-narratives: Learning to desire and create a 'fashionable' image. Teachers College Record, v.101, n.2, p. 220, 1999.

PÉREZ-SAMANIEGO, Víctor; DEVÍS-DEVÍS, José; SMITH, Brett; SPARKES, Andrew C. La investigación narrativa en la educación física y el deporte: qué es y para qué sirve. Movimento, v. 17, n.1, p.11-38, 2011.

PHOENIX, Cassandra; SPARKES, Andrew C. Young athletic bodies and narrative maps of aging. Journal of Aging Studies, v.20, n.2, p.107-121, 2006.

POLKINGHORNE, Donald E. Narrative knowing and the human sciences. Albany: Suny, 1988.

$\mathrm{RICH}$, Emma. Exploring teachers' biographies and perceptions of girls' participation in physical education. European Physical Education Review, v.10, n.2, p. 215-240, 2004.

RICHARDSON, Laurel. Writing as a method of inquiry. In: DENZIN, Norman; LINCOLN, Yvonna. The Handbook of Qualitative Research. Thousand Oaks: Sage, 1994. p. 516-529.

RICHARDSON, Laurel. New writing practices in qualitative research. Sociology of Sport Journal, v.17, n.1, p. 5-20, mar. 2000.

RODRIGUES, Renata M.; FIGUEIREDO, Zenólia C. Construção identitária da professora de Educação Física em uma instituição de educação infantil. Movimento, v. 17, n. 4, p. 65-81, 2011.

SANDERS, Clinton R.. Stranger than fiction: insights and pitfalls in post-modern ethnography. Studies in Symbolic Interaction, v.7, p. 89-104, 1995.

SARBIN, Theodore R. (Ed.). Narrative psychology. Nueva York: Praeger, 1986.

SANTOS, Núbia Z.; BRACHT, Valter; ALMEIDA, Felipe Q. Vida de Professores de Educação Física: o pessoal e o profissional no exercício da docência. Movimento, v. 15, n. 2, p. 141-165, 2009. 
SANTOS, Wagner; MAXIMIANO, Francine. Memórias discentes em Educação Física na educação básica: práticas avaliativas. Movimento, v. 19, n. 2, p. 79-101, 2013.

SANTOS, Doiara; MEDEIROS, Ana G. O futebol feminino no discurso televisivo. Revista Brasileira de Ciências do Esporte, v. 34, n. 1, p. 185-196, 2012.

SICILIA, Álvaro; FERNÁNDEZ-BALBOA, Juan-Miguel. Ethics, politics and bio-pedagogy in physical education teacher education: easing the tension between the self and the group. Sport, Education and Society, v.11, n.1, p. 1-20, 2006.

SIKES, Patricia. Growing old gracefully? Age, identity and physical education. In: EVANS, John (Ed.). Teachings, teaching and control in physical education. London: Falmer, 1988. p. $21-40$.

SMITH, Brett; SPARKES, Andrew C. Men, sport, spinal cord injury, and the construction of coherence. Narrative practice in action. Qualitative Research, v.2, p. 143-171, 2002.

SMITH, Brett; SPARKES, Andrew C. Narrative and its potential contribution to disability studies. Disability and Society, v.23, n.1, p. 17-28, 2008.

SMITH, Brett; SPARKES, Andrew C. Changing bodies, changing narratives and the consequences of tell ability: A case study of becoming disabled through sport. Sociology of Health and IIIness, v.30, n.2, p. 217-236, 2008.

SMITH, Brett; SPARKES, Andrew C. Narrative analysis and sport and exercise psychology: understanding lives in diverse ways. Psychology of Sport and Exercise, v.10, p. 279-288, 2009.

SPARKES, Andrew C. Self, silence and invisibility as a beginning teacher: A life history of lesbian experience. British Journal of Sociology of Education, v.15, n.1, p. 93-118, 1994.

SPARKES, Andrew C. Understanding teachers: A life history approach. Educational Research Monograph Series, n. 2. Exeter: School of Education, University of Exeter, 1994.

SPARKES, Andrew $C$. The fatal flaw: A narrative of the fragile body-self. Qualitative Inquiry, v.2, n.4, p. 463-494, 1996.

SPARKES, Andrew C. Recordando los cuerpos de los profesores: momentos desde el mundo de la educación física. Revista de Educación, n. 311, p. 101-122, 1996.

SPARKES, Andrew C. Ethnographic fiction and representing the absent other. Sport, Education and Society, v.2, n.1, p. 25-40, 1997.

SPARKES, Andrew C. Exploring body narratives. Sport, Education and Society, v.4, n.1, p. 17-30, 1999.

SPARKES, Andrew C. Telling tales in sport and physical activity: a qualitative journey. Champaign: Human Kinetics, 2002.

SPARKES, Andrew C.; DOUGLAS, Kitrina. Making the case for poetic representations: an example in action. Sport Psychologist, v.21, n.2, p. 170-190, 2007.

SPARKES, Andrew; NILGES, Linda; SWAN, Peter; DOWLING, Fiona. Poetic representations in sport and physical education: insider perspectives. Sport, Education and Society, v.8, n.2, p. 153-177, 2003. 
SPARKES, Andrew C.; TEMPLIN, Tom. Life histories and physical education teachers: Exploring the meanings of marginality. In: SPARKES, Andrew C. (Ed.), Research in physical education: Exploring alternative visions. London: Falmer, 1992. p. 118-145.

SQUIRE, Corinne; ANDREWS, Molly; TAMBOUKOU, Maria. Introduction: What is narrative research? In: ANDREWS, Molly; SQUIRE, Corinne; TAMBOUKOU, Maria. (Eds.). Doing narrative research. London: Sage, 2008. p. 1-21.

SQUIRES, Sara L.; SPARKES, Andrew C. Circles of silence: Sexual identity in physical education and sport. Sport, Education and Society, v.1, n.1, p. 77-101, 1996.

SYKES, Heather. Turning the closets inside/out: Towards a queer-feminist theory in women's physical education. Sociology of Sport Journal, v.15, n.2, p. 154-173, 1998.

SYKES, Heather; GOLDSTEIN, Tara. From performed to performing ethnography: Translating life history research into anti-homophobia curriculum for a teacher education program. Teaching Education, v.15, n.1, p. 41-61, 2004.

TEMPLIN, Tom; SPARKES, Andrew C.; SCHEMPP, Paul. The professional life cycle of a retired physical education teacher: A tale of bitter disengagement. Physical Education Review, v.14, n.2, p.143-155, 1991.

TEMPLIN, Tom; SPARKES, Andrew C.; GRANT, Bryan; SCHEMPP, Paul. Matching the self and aging gracefully: a life history of a late career teacher/coach. Journal of Teaching in Physical Education, v.13, n.3, p. 274-294, 1994.

WITTIZORECKI, Elisandro et al. Pesquisar exige interrogar-se: A narrativa como estratégia de pesquisa e de formação do(a) pesquisador(a). Movimento, v. 12, n.2, p. 9-33, 2006.

WRENCH, Alison; GARRETT, Robyne. Identity work: Stories told in learning to teach physical education. Sport Education and Society, v.17, n.1, p. 1-19, 2012. 